\title{
Real Hypersurfaces with Many Simple Singularities
}

\author{
Eric WeSTENBERGER \\ Universität Kaiserslautern \\ Germany \\ westenb@mathematik.uni-kl.de
}

Recibido: 22 de diciembre de 2004

Aceptado: 11 de abril de 2005

\begin{abstract}
In this paper we present constructions of real hypersurfaces with many simple singularities and deduce an asymptotical optimal existence result for hypersurfaces corresponding to T-smooth germs of the equisingular stratum. We proceed along the lines of [7] where analogous results were shown for the complex case.
\end{abstract}

Key words: simple singularities, real hypersurfaces, patchworking method.

2000 Mathematics Subject Classification: 14J17, 14J70.

\section{Introduction}

In [7] the existence of complex hypersurfaces in $\mathbb{P}^{n}=\mathbb{C P}^{n}$ with many simple isolated singularities (i.e., ADE singularities) was studied. If $S_{1}, \ldots, S_{r}$ are simple singularity types, it was shown that there exists a function $R(d) \in O\left(d^{n-1}\right)$ depending on $S_{1}, \ldots, S_{r}$ and $n$, such that the condition

$$
\sum_{i=1}^{r} k_{i} \mu\left(S_{i}\right) \leq \frac{d^{n}}{n !}-R(d)
$$

is sufficient for the existence of a reduced hypersurface $W \subset \mathbb{P}^{n}$ of degree $d$ having $k_{i}$ isolated singular points of the prescribed type $S_{i}, i=1, \ldots, r$, as its only singularities.

\footnotetext{
Work on this paper has been partially supported by the Hermann Minkowski-Minerva Center for Geometry at the Tel Aviv University and Grant No. G-616-15.6/99 from the German-Israeli Foundation for Research and Development, and by grant GR-640/9-1 DFG-Schwerpunkt "Globale Methoden in der komplexen Geometrie".
} 
Furthermore, the germ of the equisingular stratum is T-smooth at $W$, i.e., smooth of the expected codimension $\sum_{i=1}^{r} \mu\left(S_{i}\right)$ in the linear system $\left|\mathcal{O}_{\mathbb{P}^{n}}(d)\right|$. Note that $(1)$ is asymptotically optimal for hypersurfaces corresponding to T-smooth germs.

In this paper, we extend this result to real hypersurfaces in $\mathbb{R P}^{n}$. If $\mathcal{S}$ is a set of (complex) ADE singularity types, then we write $\mathcal{S}_{\mathbb{R}}$ for the set of corresponding real singularity types including pairs of complex conjugate points. For example if $\mathcal{S}=\left\{A_{1}\right\}$, then $\mathcal{S}_{\mathbb{R}}$ consists of three types: real nodes with real tangents, isolated nodes, and pairs of complex conjugate nodes. If $\bar{S}$ denotes the type of a pair of complex conjugate singular points of type $S$, we put $\mu(\bar{S})=2 \mu(S)$.

We prove that if $\mathcal{S}$ is a finite set of (complex) ADE singularity types, and $S_{1}, \ldots, S_{r}$ $\in \mathcal{S}_{\mathbb{R}}$, then the condition

$$
\sum_{i=1}^{r} k_{i} \mu\left(S_{i}\right) \leq \frac{d^{n}}{n !}-R(d), \quad R(d) \in O\left(d^{n-1}\right),
$$

is sufficient for the existence of a real, reduced hypersurface $W \subset \mathbb{R P}^{n}$ of degree $d$ having $k_{i}$ isolated singular points of type $S_{i}, i=1, \ldots, r$, no other singular points, and corresponding to a T-smooth germ.

Notice that condition (2) is again asymptotically optimal.

\section{Real topological singularity types}

Throughout this article $W$ denotes a (real or complex) hypersurface having only isolated singularities.

Let us recall some definitions and notions (see $[3,5])$.

Definition 1.1. Let $W_{1}, W_{2} \subset \mathbb{P}^{n}$ be reduced hypersurfaces, and let $z_{1}, z_{2}$ be isolated singular points of $W_{1}$, respectively $W_{2}$.

(i) The germs $\left(W_{1}, z_{1}\right),\left(W_{2}, z_{2}\right)$ are called topologically equivalent if there exists a local homeomorphism $\varphi:\left(\mathbb{P}^{n}, z_{1}\right) \rightarrow\left(\mathbb{P}^{n}, z_{2}\right)$ mapping $\left(W_{1}, z_{1}\right)$ to $\left(W_{2}, z_{2}\right)$.

If $W_{1}, W_{2}$ are defined by real polynomials, then we define

(ii) If $z_{1}, z_{2} \in \mathbb{R} \mathbb{P}^{n}$ then the germs are called topologically equivalent over $\mathbb{R}$, if the homeomorphism $\varphi$ from (i) is equivariant with respect to complex conjugation.

(iii) If $z_{1}, \overline{z_{1}}$ and $z_{2}, \overline{z_{2}}$ are pairs of conjugate imaginary singular points of $W_{1}$, respectively $W_{2}$, then the multi-germs $\left(W_{1} ; z_{1}, \overline{z_{1}}\right)$ and $\left(W_{2} ; z_{2}, \overline{z_{2}}\right)$ are called topologically equivalent over $\mathbb{R}$ if there is a local equivariant homeomorphism of multi-germs analogous to (i), (ii).

We call the equivalence classes topological types, respectively real topological types. 
Let us recall the classification of real, simple hypersurface singularities. This list can be found in several references, for example [8]. The following table lists the possible types of real, simple plane curve singularities.

\begin{tabular}{lllc} 
Type & & Normal form & (real) branches \\
\hline \hline$A_{2 k-1}^{+}$ & $(k \geq 1)$ & $x^{2}+y^{2 k}$ & 0 \\
$A_{2 k-1}^{-}(k \geq 1)$ & $x^{2}-y^{2 k}$ & 2 \\
$A_{2 k}$ & $(k \geq 1)$ & $x^{2}+y^{2 k}$ & 1 \\
\hline$D_{2 k}^{+}$ & $(k \geq 2)$ & $y\left(x^{2}+y^{2 k-2}\right)$ & 1 \\
$D_{2 k}^{-}$ & $(k \geq 2)$ & $y\left(x^{2}-y^{2 k-2}\right)$ & 3 \\
$D_{2 k+1}$ & $(k \geq 2)$ & $y\left(x^{2}+y^{2 k-1}\right)$ & 2 \\
\hline$E_{6}$ & & $x^{3}+y^{4}$ & 1 \\
$E_{7}$ & & $x\left(x^{2}+y^{3}\right)$ & 1 \\
$E_{8}$ & & $x^{3}+y^{5}$ & 1 \\
\hline \hline
\end{tabular}

Since all simple singularities have corank not bigger than 2 , the higher dimensional simple singularities are by the real splitting lemma equivalent to

$$
f\left(x_{1}, x_{2}\right) \pm x_{3}^{2} \pm \cdots \pm x_{n}^{2}
$$

where $f$ is one of the normal forms from the table above. Notice however that for example $x^{3}+y^{5}+z^{2} \sim x^{3}+y^{5}-z^{2}$, hence not all the possible normal forms in (3) yield in fact different types.

The following list shows all different types of simple surface singularities.

\begin{tabular}{lllc} 
Type & & Normal form & (real) branches \\
\hline \hline$A_{1} a$ & & $x^{2}+y^{2}+z^{2}$ & 0 \\
$A_{1} c$ & & $x^{2}+y^{2}-z^{2}$ & 2 \\
$A_{2 k-1} a$ & $(k \geq 2)$ & $x^{2}+y^{2 k}+z^{2}$ & 0 \\
$A_{2 k-1} b$ & $(k \geq 2)$ & $x^{2}-y^{2 k}+z^{2}$ & 2 \\
$A_{2 k-1} c$ & $(k \geq 2)$ & $x^{2}-y^{2 k}-z^{2}$ & 2 \\
$A_{2 k} a$ & $(k \geq 1)$ & $x^{2}+y^{2 k}+z^{2}$ & 1 \\
$A_{2 k} b$ & $(k \geq 1)$ & $x^{2}+y^{2 k}-z^{2}$ & 1 \\
\hline$D_{2 k} a$ & $(k \geq 2)$ & $y\left(x^{2}+y^{2 k-2}\right)+z^{2}$ & 1 \\
$D_{2 k} b$ & $(k \geq 2)$ & $y\left(x^{2}-y^{2 k-2}\right)+z^{2}$ & 3 \\
$D_{2 k+1} a$ & $(k \geq 2)$ & $y\left(x^{2}+y^{2 k-1}\right)+z^{2}$ & 2 \\
$D_{2 k+1} b$ & $(k \geq 2)$ & $y\left(x^{2}+y^{2 k-1}\right)-z^{2}$ & 2 \\
\hline$E_{6} a$ & & $x^{3}+y^{4}+z^{2}$ & 1 \\
$E_{6} b$ & & $x^{3}+y^{4}-z^{2}$ & 1 \\
$E_{7}$ & & $x\left(x^{2}+y^{3}\right)+z^{2}$ & 2 \\
$E_{8}$ & & $x^{3}+y^{5}+z^{2}$ & 1 \\
\hline \hline
\end{tabular}


Definition 1.2. Let $(W, z) \subset\left(\mathbb{C}^{n}, z\right)$ be an isolated hypersurface singularity locally defined by $f \in \mathbb{C}\{\mathbf{x}\}=\mathbb{C}\left\{x_{1}, \ldots, x_{n}\right\}$. Assume that $f$ is analytically equivalent to

$$
g\left(x_{1}, x_{2}\right)+x_{3}^{2}+\cdots+x_{n}^{2} .
$$

Then we define the ideal $I^{\mathrm{es}}(W, z) \subset \mathbb{C}\{\mathbf{x}\}$ to be

$$
I^{\mathrm{es}}(g)+\left\langle x_{3}, \ldots, x_{n}\right\rangle,
$$

where $I^{\mathrm{es}}(g)$ is the equisingular Tjurina ideal ([2]).

The equisingular Tjurina number of $(W, z)$ is defined by

$$
\tau^{\mathrm{es}}(W, z):=\tau^{\mathrm{es}}(g):=\operatorname{dim}_{\mathbb{C}}\left(\mathbb{C}\{\mathbf{x}\} / I^{\mathrm{es}}(g)\right) .
$$

Recall that for an ADE singularity $S$ the equisingular Tjurina number $\tau^{\mathrm{es}}(S)$ is equal to the Milnor number $\mu(S)$.

Let us introduce the real equisingular stratum. For details we refer to $[2,3,5]$.

Definition 1.3. Given real types $S_{1}, \ldots, S_{r}$ of corank $\leq 2$, let

$$
\left.V_{d}^{n}\left(S_{1}+\ldots+S_{r}\right) \subset \mid \mathcal{O}_{\mathbb{R P}^{n}}(d)\right) \mid
$$

be the (locally closed) space of all reduced hypersurfaces $W \subset \mathbb{R} \mathbb{P}^{n}$ of degree $d$ having $r$ isolated singular points $z_{1}, \ldots, z_{r}$ of types $S_{1}, \ldots, S_{r}$ as their only singularities. We call $V_{d}^{n}\left(S_{1}+\ldots+S_{r}\right)$ the real equisingular stratum.

The germ of $V_{d}^{n}\left(S_{1}+\ldots+S_{r}\right)$ at a hypersurface $W$ (or, by abuse of notation, the hypersurface itself) is called $T$-smooth if it is smooth and of the expected dimension

$$
\left(\begin{array}{c}
d+n \\
n
\end{array}\right)-1-\sum_{i=1}^{r} \tau^{\mathrm{es}}\left(S_{i}\right)
$$

Note that we have to require that the corank is $\leq 2$ since we use the topological type, which does not behave well in higher dimensions (cf. [4]).

We start by making a few general remarks concerning real singularity types:

(i) The real equisingular stratum is locally the real part of the complex equisingular stratum. This implies in particular that if the complex ES-stratum is T-smooth, then the real stratum is also T-smooth (cf. [3]).

(ii) The real topological type $S$ of a real curve singularity is determined by the $(\mu(S)+1)$-jet of a defining equation (cf. [1]).

(iii) For unibranched singularities, the real topological type is uniquely determined by the complex type since their resolution tree is a chain. For other singularities the real type depends not only on the multiplicity sequence, but also on the position of the infinitely near points (cf. [3]). 
We recall the definition of the number $\alpha_{n}^{\text {reg }}(\mathcal{S})$ from [7]:

Definition 1.4. Fix $n \geq 2$, and let $\mathcal{S}$ be a set of some real topological singularity types. Then we define $\overline{\mathcal{A}}_{n}^{\text {reg }}(\mathcal{S})$ to be the set of all $\alpha \in \mathbb{R}$ such that there exists a function $R(d) \leq \beta d^{n-1}$ with $\beta>0$, depending only on $n, \alpha$, and $\mathcal{S}$, with the property:

If for some subset $\left\{S_{1}, \ldots, S_{r}\right\} \subset \mathcal{S}$ and positive integers $k_{1}, \ldots, k_{r}$, the relation

$$
\sum_{i=1}^{r} k_{i} \tau^{\mathrm{es}}\left(S_{i}\right) \leq \alpha \cdot d^{n}-R(d)
$$

holds true, then there is a real hypersurface $W \in V_{d}^{n}\left(k_{1} S_{1}+\cdots+k_{r} S_{r}\right)$ corresponding to a $T$-smooth germ.

Put $\alpha_{n}^{\mathrm{reg}}(\mathcal{S})=\sup \mathcal{A}_{n}^{\mathrm{reg}}(\mathcal{S})$.

\section{Patchworking real singular hypersurfaces}

We construct hypersurfaces with prescribed singularities via the patchworking method for singular hypersurfaces [6]. We apply it as described in [7].

Let us briefly recall the necessary definitions and statements.

Definition 2.1. For $\Delta \subset \mathbb{R}_{+}^{n}$ compact, denote by $\mathcal{P}(\Delta) \subset \mathbb{R}[\mathbf{x}]$ the space of all real polynomials with monomials corresponding to integral points of $\Delta$.

If $F=\sum_{\omega \in \Delta \cap \mathbb{Z}^{n}} a_{\omega} \mathbf{x}^{\omega} \in \mathcal{P}(\Delta)$ and $\Delta_{+} \subset \Delta$ put

$$
F^{\Delta_{+}}:=\sum_{\omega \in \Delta_{+} \cap \mathbb{Z}^{n}} a_{\omega} \mathbf{x}^{\omega}
$$

If $F \in \mathcal{P}(\Delta)$ and $\Delta_{+} \subset \Delta$, put

$$
\mathcal{P}\left(\Delta, \Delta_{+}, F\right):=\left\{G \in \mathcal{P}(\Delta) \mid F^{\Delta_{+}}=G^{\Delta_{+}}\right\} .
$$

Definition 2.2. Let $F \in \mathbb{R}\left[x_{1}, \ldots, x_{n}\right]$ be a polynomial of degree $d$ with only isolated singular points $z_{1}, \ldots, z_{r}$ in $\left(\mathbb{C}^{*}\right)^{n}$ and whose Newton polytope $\Delta$ has dimension $n$. If $\Delta_{+}$is a union of faces of $\partial \Delta$, then we call the triple $\left(\Delta, \Delta_{+}, F\right)$ transversal if the natural map

$$
\mathcal{P}\left(\Delta, \Delta_{+}, F\right) \longrightarrow \bigoplus_{i=1}^{r} \mathcal{O}_{\mathbb{P}^{n}, z_{i}} / I^{\mathrm{es}}\left(W, z_{i}\right)
$$

is surjective, where $W$ is the hypersurface defined by $F$.

The following remark justifies the name of "transversality" (cf. [6]). 
Remark 2.3. Let $F \in \mathbb{C}[\mathbf{x}]$ be as in Definition 2.2 and let $d \geq \operatorname{deg}(F)$. Denote by $\mathcal{M}_{d}(F) \subset \mathbb{C}[\mathbf{x}]_{\leq d}$ the germ at $F$ of the equisingular stratum corresponding to the singularities of $F$ in $\left(\mathbb{C}^{*}\right)^{n}$. Then a triple $\left(\Delta, \Delta_{+}, F\right)$ as in Definition 2.2 is transversal if and only if for $d \gg 0$ the intersection

$$
\mathcal{M}_{d}(F) \cap \mathcal{P}\left(\Delta, \Delta_{+}, F\right) \subset \mathbb{C}[\mathbf{x}]_{\leq d}
$$

is transversal.

Notation 2.4. Let $\Delta_{1} \cup \ldots \cup \Delta_{N}$ be a polyhedral subdivision of a convex polytope $\Delta$. If $\Gamma$ is an orientation of the dual graph of the subdivision, define $\Delta_{k,+}, k=1, \ldots, N$, to be the union of those facets of $\Delta_{k}$ which "go into" $\Delta_{k}$ with respect to $\Gamma$, i.e., $\Delta_{k,+}=\cup_{(j, k) \in \Gamma} \Delta_{j} \cap \Delta_{k} \subset \partial \Delta_{k}$.

Theorem 2.5 (Patchworking of singular hypersurfaces). Assume we are given

- a convex subdivision $\Delta=\Delta_{1} \cup \ldots \cup \Delta_{N}$ of a convex polytope $\Delta$ of dimension $n$,

- real polynomials $F_{1}, \ldots, F_{N}$ such that

(a) $F_{k}$ has Newton polytope $\Delta_{k}$ and only isolated singular points in $\left(\mathbb{C}^{*}\right)^{n}$, $k=1, \ldots, N$,

(b) $F_{k}$ is peripherally nonsingular (PNS), i.e., for all faces $\sigma \subset \Delta_{k}$, the truncation $F^{\sigma}$ has no singular points in the torus $\left(\mathbb{C}^{*}\right)^{n}, k=1, \ldots, N$,

- subsets $\Delta_{k,+} \subseteq \partial \Delta_{k}$ given by an orientation of the dual graph of the subdivision without oriented cycles (in the sense of Notation 2.4), such that the triples $\left(\Delta_{k}, \Delta_{k,+}, F_{k}\right)$ are transversal for $k=1, \ldots, N$.

Then there exists a real polynomial $F \in \mathcal{P}(\Delta)$ such that there a bijection between the set of singular points of $F$ in $\left(\mathbb{C}^{*}\right)^{n}$ and the disjoint union of the singular points of $F_{1}, \ldots, F_{N}$ in $\left(\mathbb{C}^{*}\right)^{n}$ and the corresponding singular points have the same real topological type.

Proof. See [6].

The following theorem is the real counterpart of the stabilization theorem proven in $[7]$.

Theorem 2.6. Let $n>2$, and let $\mathcal{S}$ be a set of real topological singularity types of corank $\leq 2$ and with $\sup _{S \in \mathcal{S}} \tau^{\mathrm{es}}(S)<\infty$. For $S \in \mathcal{S}$, let $f_{S} \in \mathbb{R}\left\{x_{1}, \ldots, x_{n-1}\right\}$ be a representative of this singularity. Let $\tilde{\mathcal{S}}$ be the set of real types defined by the polynomials

$$
f_{S} \pm x_{n}^{2}, \quad S \in \mathcal{S}
$$

Then

$$
\alpha_{n}^{\mathrm{reg}}(\widetilde{\mathcal{S}}) \geq \frac{\alpha_{n-1}^{\mathrm{reg}}(\mathcal{S})}{n} .
$$

Furthermore, if $\alpha_{n-1}^{\mathrm{reg}}(\mathcal{S}) \in \mathcal{A}_{n-1}^{\mathrm{reg}}(\mathcal{S})$, then $\alpha_{n-1}^{\mathrm{reg}}(\mathcal{S}) / n \in \mathcal{A}_{n}^{\mathrm{reg}}(\widetilde{\mathcal{S}})$. 
Proof. For complex hypersurfaces the theorem was shown in [7]. The adoption to the real case is simple. If $f\left(x_{1}, \ldots, x_{n-1}\right)$ is a real PNS polynomial of degree $d-1$ having only isolated singular points. Then we consider the real polynomials

$$
F_{ \pm}\left(x_{1}, \ldots, x_{n}\right)= \pm\left(x_{n}-f-1\right)^{2}-f^{2}-2 f=0
$$

which satisfy the same properties as in the complex case and cover the two appearing real types. Then we proceed as in [7].

Note that pairs of imaginary points are kept during this construction.

\section{Constructing curves with many real simple singularities}

We shall proceed as in [7] and show that we can find polynomials defined over the real numbers with the desired singularity. After that we will deal with the existence of pairs of imaginary singular points.

\subsection{Polynomials with one (or two) simple singularities}

In the case of $A_{\mu}$-singularities, we use the quadrangles

$$
\Delta_{A_{\mu}}=\operatorname{Conv}\{(1,0),(0,1),(0,2),(\mu, 1)\},
$$

where $\operatorname{Conv}(\mathcal{A}) \subset \mathbb{R}^{2}$ denotes the convex hull of the finite set $\mathcal{A} \subset \mathbb{Z}^{2}$. Furthermore, let $\Delta_{+}=[(0,2),(0,1)] \cup[(0,1),(1,0)]$, where for $P, Q \in \mathbb{Z}^{2},[P, Q]$ is the line segment connecting $P$ and $Q$.

Lemma 3.1. Let $\mu \geq 1, \Delta=\Delta_{A_{\mu}}$.

(i) If $\mu=2 k$, then there exists an open ball $B \subset \mathcal{P}\left(\Delta_{+}\right)$such that for all $b \in B$ there exists a PNS polynomial $f \in \mathcal{P}\left(\Delta, \Delta_{+}, b\right)$ such that the complex curve defined by $f$ has a real $A_{\mu}$-singularity as its only singular point in $\left(\mathbb{C}^{*}\right)^{2}$, and the triad $\left(\Delta, \Delta_{+}, f\right)$ is transversal.

(ii) If $\mu=2 k-1$, then there exist open balls $B_{ \pm} \subset \mathcal{P}\left(\Delta_{+}\right)$such that for all $b_{ \pm} \in B_{ \pm}$ there exist PNS polynomials $f_{ \pm} \in \mathcal{P}\left(\Delta, \Delta_{+}, b_{ \pm}\right)$, such that the complex curve defined by $f_{ \pm}$has a real $A_{\mu}^{ \pm}$-singularity as its only singular point in $\left(\mathbb{C}^{*}\right)^{2}$, and the triads $\left(\Delta, \Delta_{+}, f_{ \pm}\right)$are transversal.

Proof. If $S=A_{\mu}$ we consider the polynomials

$$
f(x, y)=a y^{2}-2 y \cdot Q(x)+c x, \quad a c \neq 0, \quad \operatorname{deg} Q=\mu, \quad Q(0) \neq 0,
$$

as in the proof of $\left[7\right.$, Lemma 4.1]. For a singular point $(\alpha, \beta) \in\left(\mathbb{C}^{*}\right)^{2}$ of $f$ to be of type $A_{\mu}$, we choose a branch of $\sqrt{a c x}$ in a neighborhood of $x=\alpha$, and demand that $Q(x)-\sqrt{a c x}=(x-\alpha)^{\mu+1} \varphi(x)$, with a function $\varphi(x)$ holomorphic in a neighborhood 
of $x=\alpha$ (cf. [7]). If we choose $B$ such that $a c x>0$ around $\alpha$, we can solve this system over the real numbers as in [7].

Furthermore, the sign of $Q(0)$ determines the choice of the branch of $\sqrt{a c x}$ and hence, if $\mu=2 k-1$, the corresponding real type $A_{2 k-1}^{+}$or $A_{2 k-1}^{-}$.

For the $D_{\mu}$-singularities we use the rectangles

$$
\Delta_{D_{\mu}}:=\operatorname{Conv}\{(0,0),(\mu, 0),(\mu, 2),(0,2)\},
$$

and $\Delta_{+}=[(0,0),(0,2)] \cup[(0,0),(\mu, 0)]$.

Lemma 3.2. Let $\mu \geq 4, \Delta=\Delta_{D_{\mu}}$.

(i) If $\mu=2 k-1$, then there exists an open ball $B \subset \mathcal{P}\left(\Delta_{+}\right)$such that for all $b \in B$ there exists a PNS polynomial $f \in \mathcal{P}\left(\Delta, \Delta_{+}, b\right)$ such that the complex curve defined by $f$ has a real $D_{\mu}$-singularity as its only singular point in $\left(\mathbb{C}^{*}\right)^{2}$, and the triad $\left(\Delta, \Delta_{+}, f\right)$ is transversal.

(ii) If $\mu=2 k$, then there exist open balls $B_{ \pm} \subset \mathcal{P}\left(\Delta_{+}\right)$such that for all $b_{ \pm} \in B_{i}$ there exist PNS polynomials $f_{ \pm} \in \mathcal{P}\left(\Delta, \Delta_{+}, b_{ \pm}\right)$, such that the complex curve defined by $f_{ \pm}$has a real $D_{\mu}^{ \pm}$-singularity as its only singular point in $\left(\mathbb{C}^{*}\right)^{2}$, and the triads $\left(\Delta, \Delta_{+}, f_{ \pm}\right)$are transversal.

Proof. We consider the polynomials

$$
f(x, y)=\left(x-\alpha_{1}\right)\left(x-\alpha_{2}\right)\left(y^{2} \cdot P(x)-2 y \cdot Q(x)+R(x)\right) .
$$

If we assume that all roots of $R$ are real, then we can solve the system appearing in the proof of [7, Lemma 4.2] over the real numbers. Since we require that

$$
P R-Q^{2}=c \cdot\left(x-\alpha_{1}\right)^{\mu-2}\left(x-\alpha_{2}\right)^{\mu-2},
$$

we see that the type depends on the sign of $c$ if $\mu=2 k$. But in this case the sign of $c$ is determined by the sign of $P(0) \cdot R(0)-Q^{2}(0)$, which implies that both types can be constructed.

Remark 3.3. Real polynomials with the Newton polytopes

$$
\begin{aligned}
& \Delta_{E_{6}}=\operatorname{Conv}\{(0,0),(4,0),(0,3),(4,3)\}, \\
& \Delta_{E_{7}}=\operatorname{Conv}\{(1,0),(3,0),(3,4),(2,5),(0,5),(0,1)\}, \\
& \Delta_{E_{8}}=\operatorname{Conv}\{(0,0),(4,0),(0,4),(4,4)\},
\end{aligned}
$$

and two real $E_{6}$, respectively $E_{7}$, respectively $E_{8}$, singularities were already constructed in [7]. 


\subsection{Pairs of complex conjugate singularities}

We apply the following simple

Lemma 3.4. Let $\Delta \subset \mathbb{R}_{+}^{2}$ be a Newton polytope and let $f \in \mathcal{P}(\Delta)$ be a polynomial having singular points $z_{1}, \ldots, z_{r} \in\left(\mathbb{R}^{*}\right)^{2}$ of complex types $S_{1}, \ldots, S_{r}$ as its singular points in $\left(\mathbb{C}^{*}\right)^{2}$ with $z_{i}=\left(x_{i}, y_{i}\right)$ and $x_{i}<0$.

(i) The polynomial $F(x, y)=f\left(x^{2}, y\right)$ has $r$ pairs of complex conjugate points of type $S_{1}, \ldots, S_{r}$.

(ii) Let $T: \mathbb{Z}^{2} \rightarrow \mathbb{Z}^{2}$ be defined by $T(i, j)=(2 i, j)$ and assume that the triple $\left(\Delta, \Delta_{+}, f\right)$ is transversal, where $\Delta_{+}$is a union of faces of $\Delta$. Then the triple $\left(T(\Delta), T\left(\Delta_{+}\right), F\right)$ is also transversal.

Proof. (i) is clear.

For (ii) notice that transversality is equivalent to the fact that the coefficients of integral points of $\Delta \backslash \Delta_{+}$can be defined as smooth functions of the coefficients on $\Delta_{+} \cap \mathbb{Z}^{2}$ (cf. [7]).

\subsection{Application of the patchworking method}

The volumes of the Newton polytopes from Lemmas 3.1, 3.2, 3.4 and Remark 3.3 are always equal to the sum of Milnor numbers of the singular points of the constructed polynomials. Thus, we obtain

Proposition 3.5. Let $\left\{S_{1}, \ldots, S_{r}\right\}$ be a finite set of real, simple singularity types, and let $m=\sup _{i=1, \ldots, r}\left\{\mu\left(S_{i}\right)\right\}$. If

$$
\sum_{i=1}^{r} k_{i} \mu\left(S_{i}\right) \leq \frac{1}{2} d^{2}-m d-3
$$

then there exists a real curve $C \subset \mathbb{R P}^{2}$ of degree $d$ with $k_{i}$ singularities of type $S_{i}$, for $i=1, \ldots, r$, and no further singular points. Moreover, the real equisingular stratum is $T$-smooth at $C$.

Proof. Apply Lemmas 3.1, 3.2, 3.4 and Remark 3.3 exactly as in [7, Corollary 4.4] to obtain the result.

Corollary 3.6. If $\mathcal{S}$ is a finite set of real, simple singularity types, then

$$
\alpha_{n}^{\mathrm{reg}}(\mathcal{S})=\frac{1}{n !}
$$




\section{References}

[1] V. I. Arnol'd, S. M. Gusĕn-Zade, and A. N. Varchenko, Singularities of differentiable maps. Vol. II, Monographs in Mathematics, vol. 83, Birkhäuser Boston Inc., Boston, MA, 1988.

[2] G.-M. Greuel and C. Lossen, Equianalytic and equisingular families of curves on surfaces, Manuscripta Math. 91 (1996), no. 3, 323-342.

[3] C. Lossen, New asymptotics for the existence of plane curves with prescribed singularities, Comm. Algebra 27 (1999), no. 7, 3263-3282.

[4] I. Luengo, The $\mu$-constant stratum is not smooth, Invent. Math. 90 (1987), no. 1, 139-152.

[5] E. Shustin, Real plane algebraic curves with prescribed singularities, Topology 32 (1993), no. 4, $845-856$.

[6] __ Gluing of singular and critical points, Topology 37 (1998), no. 1, 195-217.

[7] E. Shustin and E. Westenberger, Projective hypersurfaces with many singularities of prescribed types, J. London Math. Soc. (2) 70 (2004), no. 3, 609-624.

[8] C. T. C. Wall, Deformations of real singularities, Topology 29 (1990), no. 4, 441-460. 